\title{
Ionic liquids as tunable refractive index fluids
}

\author{
Carlos Damián Rodríguez-Fernández ${ }^{1, *}$, Elena López Lago ${ }^{1}$, Christian Schröder ${ }^{2}$, and Luis M. Varela ${ }^{1}$ \\ ${ }^{1}$ Nafomat group, Departamento de Física Aplicada e Departamento de Física de Partículas, Universidade de Santiago de Compostela, \\ E-15782 Santiago de Compostela, Spain. \\ ${ }^{2}$ University of Vienna, Department of Computational Biological Chemistry, Währingerstr. 17, A-1090 Vienna, Austria.
}

\begin{abstract}
In this contribution we simulate the refractive index of several ion combinations yielding ionic liquids. The results show that their structural tunability can be exploited to successfully design liquids with task-specific refractive indices over a wide range of values, even higher than 2.0. Some designing clues are provided, being charge delocalization and the presence of fluorine atoms key factors to reach ion combinations with the highest refractive index possible.
\end{abstract}

\section{Introduction}

Ionic Liquids (ILs) are a wide family of liquid materials exclusively composed by ions which present a set of unique properties such as negligible vapour pressure, high electrochemical and thermal stability or great solvation power. However, the most important feature of ILs is that their composing ions can be independently tailored to suit specific applications. In this regard, ILs are starting to attract attention as photonic materials since their ions can be tailored to make them show fluorescence [1], liquid crystal phases [2] or thermochromism [3]. As the number of possible ion combinations is huge, computational simulations are an excellent tool to study the properties of these materials and identify the key mechanisms to tailor them. In this contribution we aim to simulate the refractive index of several combinations of IL-forming ions and to provide some designing keys to tailor the refractive index of ILs.

\section{Computational Details and Procedure}

\subsection{DFT Calculations Details}

Polarizability and volume of the ions were calculated by means of density functional theory (DFT) at the theory level B3LYP/6-311++G(d,p) using the Gaussian 16 rev. C.01 program [4]. The calculations were carried out independently for anions and cations, both in the gas phase. The optimized structures were verified by means of a vibrational analysis and the polarizability was calculated at a finite wavelength of $\lambda=589 \mathrm{~nm}$ by means of the CouplePerturbed Hartree-Fock Method (CPHF) [5].

\subsection{From ions to ionic liquids}

The polarizability of ILs, $\alpha_{I L}$ was calculated as the sum of the contributions $\alpha_{i}$ of their constituent ions $i$ : $\alpha_{I L}=\sum \alpha_{i}$.

*e-mail: damian.rodriguez@usc.es
However, their molecular volume was calculated using an empirically obtained quadratic summation rule: $V_{I L}=a_{1}$. $\sum V_{i}+a_{2} \cdot\left(\sum V_{i}\right)^{2}$ with $a_{1}=1.0189$ and $a_{2}=3.635 \times$ $10^{-4} \AA^{-3}$. The IL refractive index was calculated by means of the Lorentz-Lorenz equation:

$$
\frac{n^{2}-1}{n^{2}+2}=\frac{4 \pi}{3} \frac{\alpha_{I L}}{V_{I L}}
$$

The adimensional ratio (in cgs units), $\alpha_{I L} / V_{I L}$, of the IL is related to that of its constituent ions, $\alpha_{I L} / V_{I L} \leq \alpha_{i} / V_{i}$, which is an indicator of the individual influence of each ion in the refractive index of the IL.

\section{Studied Ions}

The considered anions are some of the most commonly used in ILs: tetrafluoroborate $[\mathrm{BF} 4]^{-}$, trisfluorotris(perfluoroethyl)phosphate $[\mathrm{FAP}]^{-}$, trifluoromethanesulfonate $[\mathrm{OTf}]^{-}$and thiocyanate $[\mathrm{SCN}]^{-}$. The first two anions contain fluorine and lack of charge delocalization while the last two present resonances. In the case of $[\mathrm{SCN}]^{-}$all the bonds participate in the resonance while it is not the case for [OTf] $]^{-}$, which also contains fluorine atoms. Regarding the cations, three families of cationic heterocycles of different alkyl chain lengths were considered: 1-alkyl-1-methylpyrrolidinium $\left[\mathrm{C}_{k} \text { mpyrr }\right]^{+}$, 1-alkyl-methylimidazolium, $\left[\mathrm{C}_{k} \mathrm{mim}\right]^{+}$, and 1-alkylpyridinium $\left[\mathrm{C}_{k} \mathrm{py}\right]^{+}$. These families of cations differ in the number of carbons in their rings, but also in their aromatic character. While in pyridinium the heterocycle is clearly aromatic, in the imidazolium the heterocycle presents a more limited conjugation and it is clearly aliphatic in pyrrolidinium. A fourth family of cations with a conjugated chain of variable length was taken into account to explicitly evaluate the influence of charge de1ocalization, 1-polyenyl-3- methylimidazolium, $\left[\mathrm{uC}_{k} \mathrm{mim}\right]^{+}$. The abbreviation and structure of all the considered ions are shown in Table 1. 
Table 1. IL-forming ions studied in this work

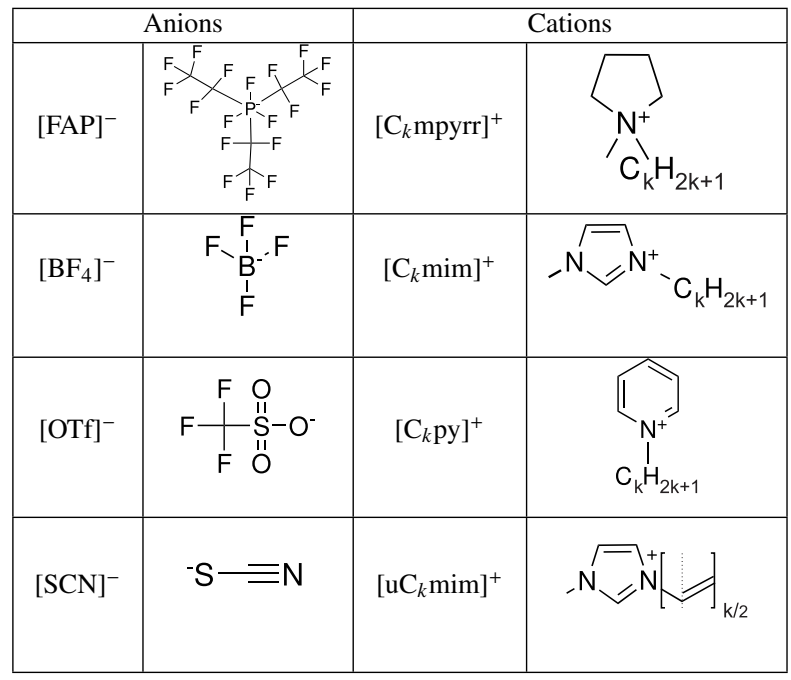

\section{Results}

\subsection{Anionic Influence}

The $\alpha_{i} / V_{i}$ ratio is very different for the different anions studied due to their large structural and compositional differences. Table 2 shows the ratios of selected anions.

Table 2. $\alpha_{i} / V_{i}$ ratio of selected anions.

\begin{tabular}{|l|c|}
\hline Anion & $\alpha_{i} / V_{i}$ \\
\hline$\left[\mathrm{BF}_{4}\right]^{-}$ & 0.041 \\
\hline$[\mathrm{FAP}]^{-}$ & 0.050 \\
\hline$[\mathrm{OTf}]^{-}$ & 0.061 \\
\hline$[\mathrm{SCN}]^{-}$ & 0.086 \\
\hline
\end{tabular}

Inorganic anions without electronic charge delocalization and including fluorine atoms such as $\left[\mathrm{BF}_{4}\right]^{-}$or $[\mathrm{FAP}]^{-}$present the lowest ratios. The presence of fluorine atoms in a molecule is expected to decrease its overall polarizability since highly electronegative atoms produce tightly bonded electronic densities which are hardly polarizable. As a consequence, these anions are expected to provide low refractive index ILs. In contrast, those anions showing resonance due to charge delocalization throughout their $\pi$ bonds such as [OTf] ${ }^{-}$or $[\mathrm{SCN}]^{-}$show higher $\alpha_{i} / V_{i}$ ratios. Among them, the $[\mathrm{SCN}]^{-}$anion, with all its bonds participating in the resonance, presents the highest one. In fact, the value of this ratio for [OTf] $]^{-}$is much more moderate than for $[\mathrm{SCN}]^{-}$, since not all the bonds in the molecule participate in the resonance and there are fluorine atoms in its molecular structure. Hence, among the considered anions, the most promising candidate to yield high refractive index ILs is the $[\mathrm{SCN}]^{-}$.

\subsection{Cationic Influence}

The $\alpha_{i} / V_{i}$ ratio of the considered cations for two different chain lengths, $(k=2)$ and $(k=5)$, is shown in Table 3. For a fixed chain length, $k=5$, the $\alpha_{i} / V_{i}$ ratio increases with conjugation: $\left[\mathrm{C}_{k} \text { mpyrr }\right]^{+}<\left[\mathrm{C}_{k} \mathrm{mim}\right]^{+}<$
Table 3. $\alpha_{i} / V_{i}$ ratio of considered cations.

\begin{tabular}{|l|c|c|}
\hline Cation & $\alpha_{i} / V_{i}(k=2)$ & $\alpha_{i} / V_{i}(k=5)$ \\
\hline$\left[\mathrm{C}_{k} \mathrm{mpyrr}\right]^{+}$ & 0.066 & 0.067 \\
\hline$\left[\mathrm{C}_{k} \mathrm{mim}\right]^{+}$ & 0.070 & 0.070 \\
\hline$\left[\mathrm{C}_{k} \mathrm{py}\right]^{+}$ & 0.074 & 0.073 \\
\hline$\left[\mathrm{uC}_{k} \mathrm{mim}\right]^{+}$ & 0.076 & 0.100 \\
\hline
\end{tabular}

$\left[\mathrm{C}_{k} \mathrm{py}\right]^{+}<\left[\mathrm{uC}_{k} \mathrm{mim}\right]^{+}$. Hence, higher refractive indices are expected for ILs with more extensive regions of conjugation. This is an unequivocal evidence of the large influence of charge delocalization in the polarizability of ILforming ions. With respect to the side chain variation, its elongation produces different effects in heterocycles bearing alkyl chains or conjugated chains. When an alkyl chain is varied, it produces small changes in the $\alpha_{i} / V_{i}$ ratio, which could be used to finely tune the refractive index of derived ILs. However, when this variation is done in a polyenyl conjugated chain, the effect on polarizability is much more drastic than that observed in saturated alkyl chains. In order to shed more light on this, we show in Fig. 1 the polarizability as a function of the chain length for both $\left[\mathrm{C}_{k} \mathrm{mim}\right]^{+}$and $\left[\mathrm{uC}_{k} \mathrm{mim}\right]^{+}$families, which only differ in the conjugated character of their chains.

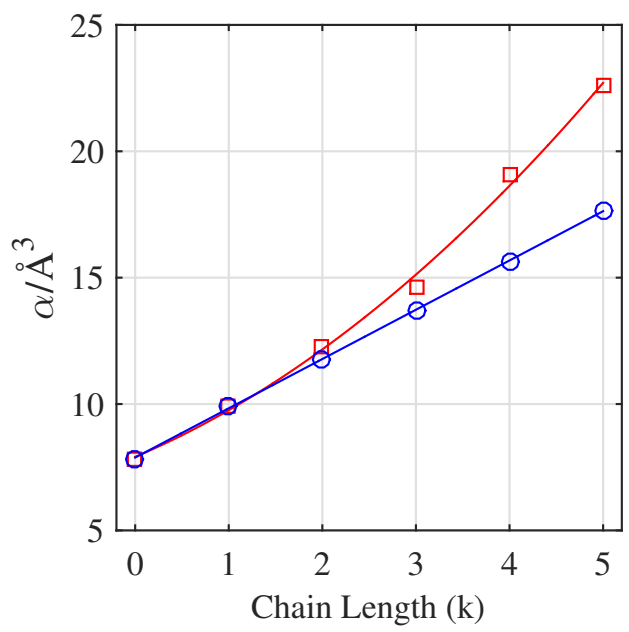

Figure 1. Electronic polarizability of $\left[\mathrm{C}_{k} \mathrm{mim}\right]^{+}$(०) and $\left[\mathrm{uC}_{k} \mathrm{mim}\right]^{+}(\square)$ cation families as function of the chain length.

The figure reveals that polarizability is a linear function of the saturated chain length but a supra-linear function of the conjugated one. According to theory [6], polarizability is proportional to $L^{4}$, the fourth power of the physical extension where electrons are allowed to move. In the saturated case, electrons are highly localized in the $\sigma$ bonds of each $-\mathrm{CH}_{2}-$ unit. Hence, adding new units to the alkyl chain produces a linear increase of polarizability since the contribution of the $-\mathrm{CH}_{2}-$ unit is $k$ times independently added to that of the imidazolium heterocycle. On the other hand, in the case of the conjugated unsaturated chain, the electrons of each $=\mathrm{CH}_{2}-$ unit partic- 
ipate in the charge delocalization that takes place all over the conjugated $\pi$ bonds of the side chain. Thus, in this case, with each new bond added to the side chain, the effective length where electrons are allowed to move in the molecule is enlarged $(L)$, producing the observed nonlinear increase of polarizability. According to this phenomenology, the most suitable cations to produce ILs with high refractive index are those that present large aromaticity in their heterocycles, such as $\left[\mathrm{C}_{k} \mathrm{py}\right]^{+}$, or in their side chains, such as those belonging to the $\left[\mathrm{uC}_{k} \mathrm{mim}\right]^{+}$family.

\subsection{Ionic Liquids}

The refractive index of the combination of all the anions with those cations of chain length $k=2$ and $k=5$ are shown in Table 4. With only the considered ionic species, it is possible to synthesize ILs with refractive indices ranging from 1.36 to 1.69 . The lowest values correspond to combinations of ions lacking of charge delocalization and containing fluorine atoms. On the other hand, the highest refractive indices belong to combinations where both ions present charge delocalization and lack of fluorine.

Table 4. Refractive index of ILs with cations bearing side chains of length $k=2$ (first value) and $k=5$ (second value).

\begin{tabular}{|l|c|c|c|c|}
\hline & \multicolumn{4}{|c|}{ Anions } \\
\hline Cations & {$[\mathrm{FAP}]^{-}$} & {$\left[\mathrm{BF}_{4}\right]^{-}$} & {$[\mathrm{OTf}]^{-}$} & {$[\mathrm{SCN}]^{-}$} \\
\hline$\left[\mathrm{C}_{k} \mathrm{mpyrr}\right]^{+}$ & $1.36-1.37$ & $1.39-1.41$ & $1.43-1.43$ & $1.50-1.49$ \\
\hline$\left[\mathrm{C}_{k} \mathrm{mim}\right]^{+}$ & $1.37-1.38$ & $1.40-1.42$ & $1.44-1.45$ & $1.52-1.51$ \\
\hline$\left[\mathrm{C}_{k} \mathrm{py}\right]^{+}$ & $1.38-1.39$ & $1.42-1.45$ & $1.46-1.47$ & $1.54-1.53$ \\
\hline$\left[\mathrm{uC}_{k} \mathrm{mim}\right]^{+}$ & $1.39-1.46$ & $1.44-1.59$ & $1.47-1.60$ & $1.55-1.69$ \\
\hline
\end{tabular}

As shown in the Table, the presence of delocalized charge regions and avoiding fluorine atoms are the key features to produce ILs with very high refractive index. In this regard, it is possible to perform extrapolations based on our calculations to predict the evolution of the refractive index as a function of the side chain length of the most promising cations, $\left[\mathrm{C}_{k} \mathrm{py}\right]^{+}$and $\left[\mathrm{uC}_{k} \mathrm{mim}\right]^{+}$ together with the most promising anion $\left[\mathrm{SCN}^{-}\right.$. Fig. 2 shows the evolution of $\left[\mathrm{C}_{k}\right.$ py] $[\mathrm{SCN}]$ and $\left[\mathrm{uC}_{k} \mathrm{mim}\right][\mathrm{SCN}]$ families of ILs as a function of the chain length from $k=2$ to $k=16$. At the shortest chain lengths, $k=0,1$, the $\left[\mathrm{C}_{k} \mathrm{py}\right][\mathrm{SCN}]$ family yields higher refractive indices than $\left[\mathrm{uC}_{k} \mathrm{mim}\right][\mathrm{SCN}]$ one, which is a consequence of the higher aromaticity of the pyridinium heterocycle in comparison with imidazolium. However, when increasing the chain length, the refractive index of the $\left[\mathrm{C}_{k} \mathrm{py}\right][\mathrm{SCN}]$ family decreases while the refractive index of $\left[\mathrm{uC}_{k} \mathrm{mim}\right][\mathrm{SCN}]$ monotically increases. In the case of $\left[\mathrm{C}_{k}\right.$ py $][\mathrm{SCN}]$ the low polarizability of the alkyl chain in comparison with that of the pyridinium heterocycle and the thiocyanate anion is the cause of the overall decreasing of the refractive index. On the other hand, as conjugated chains non-linearly increase their polarizability with their length, increasing the side chain in $\left[\mathrm{uC}_{k} \mathrm{mim}\right][\mathrm{SCN}]$ leads to an overall increasing of refractive index, which reaches the threshold of 2.0 with $k=11$.

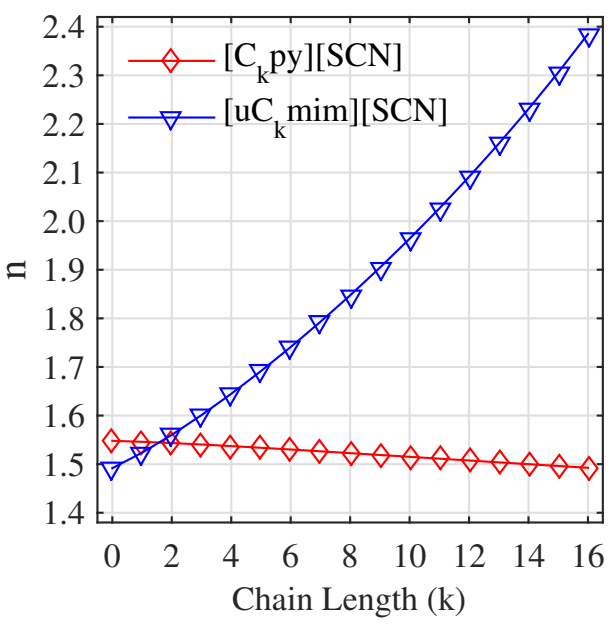

Figure 2. Refractive index extrapolation of $\left[\mathrm{C}_{k}\right.$ py] $[\mathrm{SCN}](\diamond)$ and $\left[\mathrm{uC}_{k} \mathrm{mim}\right][\mathrm{SCN}](\nabla)$ families of ILs as function of the cation side chain length.

\section{Conclusions}

ILs are materials with an inherent large degree of tunability and potential applications in photonics. Wise selection of IL's constituents provides refractive index fluids covering a wide range of refractive indices, from 1.36 to 1.69 . Charge delocalization and presence of fluorine atoms are key aspects to produce combinations in both upper and lower limits of the previous range. According to our calculations, increasing the degree of charge delocalization in cations could provide ILs with refractive indices higher than 2.0, such as in [SCN] $\left[\mathrm{uC}_{k} \mathrm{mim}\right]$.

\section{References}

[1] K. Lunstroot, K. Driesen, P. Nockemann, C. GörllerWalrand, K. Binnemans, S. Bellayer, J. Le Bideau, A. Vioux, Chem. Mater. 18, 5711 (2006)

[2] K. Tanabe, Y. Suzui, M. Hasegawa, T. Kato, J. Am. Chem. Soc. 134, 5652 (2012)

[3] S.J. Osborne, S. Wellens, C. Ward, S. Felton, R.M. Bowman, K. Binnemans, M. Swadźba-Kwäny, H.Q. Nimal Gunaratne, P. Nockemann, Dalt. Trans. 44, 11286 (2015)

[4] M.J. Frisch, G.W. Trucks, H.B. Schlegel, G.E. Scuseria, M.a. Robb, J.R. Cheeseman, G. Scalmani, V. Barone, G.a. Petersson, H. Nakatsuji et al., Gaussian 16, Revision C.01 (2016)

[5] J. Dodds, R. McWeeny, W. Raynes, J. Riley, Mol. Phys. 33, 611 (1977)

[6] K. Rustagi, J. Ducuing, Opt. Commun. 10, 258 (1974) 